\title{
Identification of key genes associated with Schmid-type metaphyseal chondrodysplasia based on microarray data
}

\author{
BING WANG ${ }^{1}$, LI HE ${ }^{2}$, WUSHENG MIAO ${ }^{1}$, GE WU ${ }^{1}$, HAI JIANG ${ }^{1}$, YONGTAO WU ${ }^{1}$, JINING QU ${ }^{1}$ And MIN LI ${ }^{1}$ \\ ${ }^{1}$ Department of Pediatric Orthopedics, Honghui Hospital, Xi'an Jiaotong University, Xi'an, Shaanxi 710054; \\ ${ }^{2}$ Department of Child Health Care, Xi'an Children's Hospital, Xi'an, Shaanxi 710003, P.R. China
}

Received November 11, 2015; Accepted March 28, 2017

DOI: $10.3892 /$ ijmm.2017.2954

\begin{abstract}
This study aimed to gain a better understanding of the molecular circuitry of Schmid-type metaphyseal chondrodysplasia (SMCD), and to identify more potential genes associated with the pathogenesis of SMCD. Microarray data from GSE72261 were downloaded from the NCBI GEO database, including collagen $X$ p.Asn617Lys knock-in

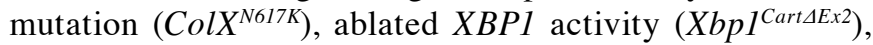
compound mutant $(\mathrm{C} / \mathrm{X})$, and wild-type (WT) specimens. Differentially expressed genes (DEGs) were screened in Xbp1 vs. WT, Col vs. WT and CX vs. WT, respectively. Pathway enrichment analysis of these DEGs was performed. Transcription factors (TFs) of the overlapping DEGs were identified. Weighted correlation network analysis (WGCNA) was performed to find modules of DEGs with high correlations, followed by gene function analysis and a protein-protein interaction network construction. In total, 481, 1,530 and 1,214 DEGs were identified in Xbp1 vs. WT, Col vs. WT and CX vs. WT, respectively. These DEGs were enriched in different pathways, such as extracellular matrix (ECM)receptor interaction and metabolism-related pathways. A total of 7 TFs were found to regulate 19 common upregulated genes, and 4 TFs were identified to regulate 21 common downregulated genes. Two significant gene co-expression modules were enriched and DEGs in the 2 modules were mainly enriched in different biological processes, such as ribosome biogenesis. Moreover, Kras (downregulated), Col5al (upregulated) and Furin (upregulated) were both identified in the regulatory networks and protein-protein interaction (PPI) network. On the whole, our findings indicate that the Kras, Col5al and Furin genes may play essential roles in the molecular mechanisms of SMCD, which warrants further investigation.
\end{abstract}

Correspondence to: Dr Li He, Department of Child Health Care, Xi'an Children's Hospital, 69 West Juyuan Alley, Lianhu, Xi'an, Shaanxi 710003, P.R. China

E-mail: heliihhhhh@hotmail.com

Key words: Schmid-type metaphyseal chondrodysplasia, differentially expressed genes, functional enrichment analysis, protein-protein interaction network

\section{Introduction}

Schmid-type metaphyseal chondrodysplasia (SMCD), a relatively common form of metaphyseal chondrodysplasia, is an autosomal inherited chondrodysplasia; affected patients are of short stature and exhibit coxa vara, genu varum and a waddling gait due to skeletal deformities, resulting from growth plate cartilage abnormalities $(1,2)$. Although chondrodysplasias are considered rare [the incidence of chondrodysplasias is $1 / 4,000$ births (3)], they severely affect the quality of life of affected individuals. Chondrodysplasias have diverse etiologies and there is ample evidence to indicate that SMCD is caused by heterozygous mutations in the gene of collagen, type X, alpha 1 (CollOal) (4). The gene encodes a chain of type $X$ collagen molecule whose expression is largely restricted to zones of calcifying or degrading growth plate cartilage, and thus the mutations of CollOal may interfere with endochondral ossification (5).

It has been demonstrated that many mutations in genes that encode cartilage extracellular matrix (ECM) molecules are involved in the etiology of chondrodysplasias (6). Chondrodysplasias caused by mutations associated with ECM are due to inappropriate processing, folding, or export of the abnormal ECM molecules, resulting in the accumulation of these molecules within the endoplasmic reticulum (ER) of chondrocytes $(3,7)$. Subsequent to the cellular retention of mutant ECM proteins, chondrocyte ER stress and the unfolded protein response (UPR) are activated, in an attempt by the cells to deal with the inappropriate retention of mutant proteins (8). Rajpar et al used the mouse models phenocopying SMCD, a condition involving dwarfism and hypertrophic zone expansion of the growth plate caused by autosomal dominant mutations in CollOal, and demonstrated the central importance of ER stress in the pathology of SMCD (9). By contrast, the UPR can be activated via ER membrane-spanning sensors, such as inositolrequiring enzyme-1 (IRE1) and activated IRE1 can splice the coding sequence of the $\mathrm{X}$-box binding protein 1 (XBPl), a transcription factor (TF) responsible for multiple UPR target gene expression (10). Recently, Cameron et al demonstrated that the IRE1/XBP1 pathway was redundant to cartilage pathology and they proposed that the XBP1-independent UPR-driven dysregulation of CCAAT/enhancer binding protein $\beta$ (C/EBP- $\beta)$, a TF important for the transition from chondrocyte proliferation to hypertrophy, was significant for the pathophysiology of 
SMCD (11). However, the limited studies have not yet revealed the details of the molecule circuitry in SMCD.

In this study, we downloaded the microarray data from GSE72261, the same gene expression profiling used in the study by Cameron et al, from a publicly available database (11). Mice with a SMCD collagen X p.Asn617Lys knock-in mutation $\left(\mathrm{ColX}^{N 617 K}\right)$ were crossed with mice in which $X B P 1$ activity was ablated specifically in cartilage $\left(X b p 1^{\operatorname{Cart} \Delta E x 2}\right)$, generating the compound mutant, $\mathrm{C} / \mathrm{X}$ (11). In the study by Cameron et al (11), they mainly focused on the analysis of role of the IRE1/XBP1 pathway in the pathophysiology of SMCD, and thus they represented the XBP1-dependent gene expression changes using differentially expressed probes between $\operatorname{ColX}^{N 617 K}$ and wild-type (WT), but not between $X b p 1^{\text {Cart } \Delta E x 2}$ and WT or C/X and WT; the XBP1-independent gene expression changes using differentially expressed probes between $\operatorname{Col}^{N 617 K}$ and WT, and between $\mathrm{C} / \mathrm{X}$ and WT, but not $X b p 1^{\operatorname{Cart} \Delta E x 2}$ and WT. However, they did not analyze the gene part between $\operatorname{Col}^{N 617 K}$ and WT,

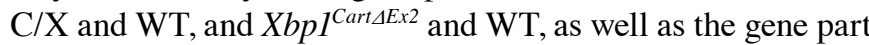
between $\operatorname{ColX}^{N 617 K}$ and WT, $X b p 1^{\text {Cart } \Delta E x 2}$ and WT, but not C/X and WT.

In the present study, differentially expressed genes (DEGs) were screened in $\mathrm{ColX}^{N 617 K}, \mathrm{Xbpl}^{\mathrm{Cart} \Delta E x 2}$ and $\mathrm{C} / \mathrm{X}$ hypertrophic zone samples compared with WT hypertrophic zone specimens, respectively. Subsequently, comprehensive bioinformatics was used to analyze the significant pathways of the DEGs, identification of TFs of the overlapping DEGs both in the three comparison groups and construction of the corresponding regulation networks. Furthermore, to further analyze the more potential genes associated with SMCD, gene co-expression modules, as well as protein-protein interaction (PPI) networks were constructed based on the sum of DEGs. We focused on the overlap of the results between regulatory networks and co-expression analysis. The aim of this study was to gain a better understanding of the molecular circuitry of SMCD, and to identify more potential genes associated with the pathogenesis of SMCD.

\section{Materials and methods}

Microarray data and data preprocessing. The microarray data of GSE72261, deposited by Cameron et al (11), were downloaded from the National Center for Biotechnology Information (NCBI) Gene Expression Omnibus (GEO) database (http://www.nibi. nih.gov/geo/). As described in the original study (11), hypertrophic zones were microdissected from one proximal tibial growth plate from three 2-week old WT mice, three 2-week old mice carrying a Colloal p.N617K mutation $\left(\right.$ Col $\left.^{N 617 K}\right)$, three 2-week old mice lacking XBP1 activity in chondrocytes $\left(X b p 1^{\text {Cart } \triangle E x 2}\right)$, and three 2-week old mice resulting from a cross between $\operatorname{ColX}^{N 617 K}$ and $X b p 1^{\operatorname{Cart} \Delta E x 2}(\mathrm{C} / \mathrm{X})$. Thus, the GSE72261 dataset consisted of 3 WT samples, $3 \mathrm{Xbp}^{\mathrm{Cart} \Delta \mathrm{Ex} 2}$ specimens (named Xbp1), $3 \mathrm{ColX}^{\mathrm{N} 617 \mathrm{~K}}$ specimens (named $\mathrm{Col}$ ) and $3 \mathrm{C} / \mathrm{X}$ specimens (named $\mathrm{C} / \mathrm{X}$ ). The corresponding platform was GPL6887 Illumina MouseWG-6 v2.0 expression beadchip. In this study, all these 12 samples were selected to carry out the follow-up analysis.

The non-normalized data were downloaded and preprocessed using preprocessCore package (12) which is a part of Bioconductor project (http://www.bioconductor.org/). Besides, with the use of org.Mm.eg.db (13) and illuminaMousev2.db (14) packages of bioconductor, the probe symbols were transformed into corresponding gene symbols. The expression value was averaged for each gene when multiple probes were mapped to the same gene. After data preprocessing, 20,106 gene expression matrix were received.

Screening of DEGs. The DEGs in Xbp1 vs. WT, Col vs. WT, and CX vs. WT were analyzed using the limma package (15) in $\mathrm{R} /$ Bioconductor. $\log _{2}$ fold change $(\mathrm{FC}) \mathrm{I}$ and p-values from an unpaired t-test implemented in the limma package (15) were used to select the DEGs. Values of $\mathrm{p}<0.01$ and $\mid \log _{2} \mathrm{FCl} \geq 0.58$ (showing $>1$.5-fold differential expression) were set as the cut-off criteria. Additionally, the DEGs identified in Xbp1 vs. WT, Col vs. WT and CX vs. WT, respectively were clustered using the gplots package (16) in R, evaluating whether the DEGs identified were sample-specific. The results were displayed as heatmaps.

By contrast, in the original study by Cameron et al (11), DEGs were screened in Xbp1 vs. WT, Col vs. WT and CX vs. WT, respectively with $>2$-fold differential expression and with an adjusted p-value of 0.01. Moreover, Cameron et al focused on the DEGs in Col vs. WT, but not Xbpl vs. WT or CX vs. WT, and the DEGs in Col vs. WT and CX vs. WT, but not Xbp1 vs. WT, in the subsequent analysis.

Pathway enrichment analysis. The database for annotation, visualization and integrated discovery (DAVID) online software can provide a comprehensive set of functional annotation tools (17). In order to analyze the identified DEGs on the functional level, the Kyoto Encyclopedia of Genes and Genomes (KEGG) pathway enrichment analysis was performed for all DEGs using DAVID software (17). Subsequently, p-values were calculated using the hypergeometric test (18). Gene count $\geq 2$ and p-values $<0.05$ were set as the cut-off criterion for pathway enrichment analysis.

Identification of TFs and construction of the regulatory network. iRegulon (19), available as a Cytoscape (20) plugin, implements a genome-wide ranking-and-recovery approach to detect enriched TF motifs and their optimal sets of direct target genes (19). Besides, iRegulon allows the integration of predicted regulatory binding sites directly into a biological network (19). In the original study by Cameron et al (11), DEGs in Col vs. WT and CX vs. WT and Xbp1 vs. WT, as well as the DEGs between in Col vs. WT and Xbp1 vs. WT, but not CX vs. WT, were analyzed. In this study, we screened out the overlapping DEGs with consistent expression changes (both upregulated or both downregulated) in the 3 groups (Xbp1 vs. WT, Col vs. WT and CX vs. WT). The lists of overlapping DEGs with consistent expression change were subjected to iRegulon (19) and used to predict their transcriptional regulators using the following parameters: minimum identity between orthologous genes, 0.05; and maximum false discovery rate on motif similarity, 0.001. The predicted TF-DEG pairs with normalized enrichment scores (NES) (19) $>4.5$ were selected for further analysis and the regulatory networks were constructed.

Analysis of gene co-expression modules. To further analyze the more potential genes associated with SMCD, we intended 
to perform gene co-expression analysis of DEGs. For reducing the deviation, we used all the DEGs as the scope of gene co-expression analysis.

Weighted correlation network analysis (WGCNA) can be used for finding modules (clusters) of genes with high correlations, or relating modules to external sample traits (21). A robust correlation coefficient emphasizes high correlations of genes and results in a weighted network (21). In this study, WGCNA R software package (21), which can cluster the most highly co-expressed genes in defined modules, was applied to detect modules of co-expressed genes of all the DEGs identified in Xbp1 vs. WT, Col vs. WT, and CX vs. WT. If the absolute value of the correlation coefficient was high, the co-expressed genes clustered in modules would have high gene co-expression trend consistency and the modules would be significantly related to external sample traits.

Functional enrichment analysis of genes in the co-expression modules. In order to analyze gene co-expression modules on the functional level, Gene Ontology (GO) enrichment analysis was carried out using DAVID online tool (17) to obtain the enriched biological process (BP) terms. A hypergeometric test (18) was applied to examine the significance of this enrichment analysis. The count number $\geq 2$ and $p$-value $<0.05$ were used as the cut-off criterion.

Construction of PPI network based on genes in the co-expression modules. The Search Tool for the Retrieval of Interacting Genes (STRING) database can be used as it provides easy access to known and predicted protein interactions (22). The interaction probabilities of proteins in STRING are provided with a confidence score (22). A protein with a confidence score $>0.4$ is deemed to have medium confidence of interaction with other proteins (23). In the present study, the STRING database was used to select the PPIs among the DEGs identified in the co-expression modules with default parameters (species: mus musculus). Interaction pairs of DEGs with the confidence score $\geq 0.4$ were selected for the PPI network construction and the network was visualized using Cytoscape software (24), where nodes indicated proteins and edges indicated physical interactions. In addition, node degrees of the DEGs were calculated. The nodes with higher degree in the network were considered as hub proteins.

Furthermore, we integrated the results of regulatory networks and PPI networks. The DEGs identified both in the regulatory network and PPI network may be more potential key genes associated with SMCD.

\section{Results}

Screening of DEGs. According to the gene expression profile, with p-values $<0.01$ and $\mid \log 2 \mathrm{FCl} \geq 0.58$, there were 481 DEGs identified in Xbpl vs. WT, comprising 308 upregulated genes and 173 downregulated genes. The corresponding heatmap of the DEGs is shown in Fig. 1A. Furthermore, 1,530 DEGs (831 up- and 699 downregulated genes) and 1,214 DEGs (640 up- and 574 downregulated genes) were screened out in Col vs. WT and CX vs. WT, respectively. The respective heatmaps are shown in Fig. 1B and C. From the heatmaps, we found that the identified DEGs could distinguish the experimental samples from the WT samples, suggesting the DEGs were eligible for the subsequent analysis.

KEGG pathway enrichment analysis of the DEGs identified. The KEGG pathways of the significantly upregulated and downregulated DEGs are summarized in Table I. In total, 6 pathways were enriched based on the DEGs identified in Xbp1 vs. WT, such as ECM-receptor interaction and focal adhesion. Moreover, a number of pathways were enriched by the upregulated and downregulated genes in Col vs. WT and CX vs. WT. Table I only displays the top 10 and 5 pathways enriched by these DEGs in Col vs. WT and CX vs. WT, respectively. For instance, the upregulated genes in Col vs. WT were significantly associated with aminoacyl-tRNA biosynthesis and associated with ER stress (11), such as RNA degradation and glutathione metabolism. The downregulated genes in Col vs. WT were associated with focal adhesion. On the other hand, the upregulated genes identified in CX vs. WT were significantly associated with glycolysis/gluconeogenesis, as well as with the fructose and mannose metabolism pathways. The downregulated genes in CX vs. WT were found to be linked with leukocyte transendothelial migration.

Analysis of the regulatory network. Among all the DEGs identified in Xbp1 vs. WT, Col vs. WT and CX vs. WT, a total of 24 common upregulated genes and 43 common downregulated genes were identified. The expression changes of these common DEGs in all the samples are shown in the heatmap (Fig. 1D). With NES $>4.5$, total 7 TFs were found to regulate 19 common upregulated genes (Fig. 2A). These 7 TFs were DEAF1 transcription factor (Deaf1, NES=5.608), sterol regulatory element binding transcription factor 1 (Srebf1, NES=5.579), GATA binding protein 1 (Gata1, NES=5.315), Hes Family BHLH transcription factor 5 (Hes5, NES=5.135), RAR-related orphan receptor C (Rorc, NES=4.74), hedgehog acyltransferase (Hhat, $\mathrm{NES}=4.593$ ) and CAMP responsive element binding protein 3-like 1 (Cereb311, NES=4.535). By contrast, a total of 4 TFs were identified to regulate 21 common downregulated genes (Fig. 2B). These 4 TFs were zinc finger and BTB domain containing 14 (Zbtb14, NES=5.722), SMAD family member $4(\operatorname{Smad} 4, \mathrm{NES}=5.313)$, TATA box binding protein (Tbp, NES=5.137), JAZF zinc finger 1 (Jazf1, $\mathrm{NES}=4.627$ ). However, all the TFs that could regulate upregulated genes or downregulate genes were not DEGs.

Gene co-expression modules and functional enrichment analysis of genes in these modules. As already indicated, all the identified DEGs in the 3 groups (Xbpl vs. WT, Col vs. WT and CX vs. WT) were used to construct the co-expression network. Thus, 2,258 genes were included (967 common genes were removed, including overlapping DEGs in the 3 groups or only 2 groups). WGCNA analysis identified a total of 11 modules with highly co-expressed genes (Fig. 2C). Besides, this analysis led to the identification of dark green-colored (correlation coefficient, 0.94) and green-colored (correlation coefficient, -0.89) modules with highest significance value (Table II). The results of several significant GO BP terms (ranked by p-value) of DEGs in these 2 colored modules are shown in Table III. The results demonstrated that genes enriched in the dark green-colored module were significantly associated with the biological 
Table I. The pathways enriched in the Xbp1 group, and the top 10 pathways and top 5 pathways enriched in the Col group and CX group, respectively.

\begin{tabular}{|c|c|c|c|}
\hline Gene change & KEGG term & Count & p-value \\
\hline \multicolumn{4}{|l|}{ In Xbp1 group } \\
\hline \multirow[t]{4}{*}{ Upregulated } & mmu04512:ECM-receptor interaction & 8 & $5.23 \mathrm{E}-04$ \\
\hline & mmu04510:Focal adhesion & 11 & 0.0020 \\
\hline & mmu04142:Lysosome & 8 & 0.0043 \\
\hline & mmu00561:Glycerolipid metabolism & 4 & 0.0468 \\
\hline \multirow[t]{2}{*}{ Downregulated } & mmu03010:Ribosome & 8 & $2.69 \mathrm{E}-05$ \\
\hline & mmu00860:Porphyrin and chlorophyll metabolism & 3 & 0.0364 \\
\hline \multicolumn{4}{|l|}{ In Col group } \\
\hline \multirow[t]{10}{*}{ Upregulated } & mmu00970:Aminoacyl-tRNA biosynthesis & 8 & 0.0011 \\
\hline & mmu00051:Fructose and mannose metabolism & 7 & 0.0030 \\
\hline & mmu00600:Sphingolipid metabolism & 7 & 0.0057 \\
\hline & mmu00250:Alanine, aspartate and glutamate metabolism & 6 & 0.00594 \\
\hline & mmu04142:Lysosome & 12 & 0.0071 \\
\hline & mmu00290:Valine, leucine and isoleucine biosynthesis & 4 & 0.00796 \\
\hline & mmu00510:N-Glycan biosynthesis & 7 & 0.00893 \\
\hline & mmu03018:RNA degradation & 8 & 0.00895 \\
\hline & mmu00480:Glutathione metabolism & 7 & 0.0160 \\
\hline & mmu00010:Glycolysis/gluconeogenesis & 8 & 0.0172 \\
\hline \multirow[t]{10}{*}{ Downregulated } & mmu04510:Focal adhesion & 24 & $1.18 \mathrm{E}-05$ \\
\hline & mmu04070:Phosphatidylinositol signaling system & 14 & $1.55 \mathrm{E}-05$ \\
\hline & mmu04142:Lysosome & 15 & $5.58 \mathrm{E}-04$ \\
\hline & mmu04270:Vascular smooth muscle contraction & 15 & $6.08 \mathrm{E}-04$ \\
\hline & mmu04512:ECM-receptor interaction & 12 & 8.03E-04 \\
\hline & mmu04912:GnRH signaling pathway & 13 & $8.79 \mathrm{E}-04$ \\
\hline & mmu05210:Colorectal cancer & 12 & 0.00108 \\
\hline & mmu04540:Gap junction & 12 & 0.00108 \\
\hline & mmu05213:Endometrial cancer & 9 & 0.00157 \\
\hline & mmu04670:Leukocyte transendothelial migration & 14 & 0.00175 \\
\hline \multicolumn{4}{|l|}{ In $\mathrm{CX}$ group } \\
\hline \multirow[t]{5}{*}{ Upregulated } & mmu00010:Glycolysis/gluconeogenesis & 9 & 0.0012 \\
\hline & mmu00051:Fructose and mannose metabolism & 6 & 0.0055 \\
\hline & mmu00030:Pentose phosphate pathway & 5 & 0.0082 \\
\hline & mmu00520:Amino sugar and nucleotide sugar metabolism & 6 & 0.0116 \\
\hline & mmu00750:Vitamin B6 metabolism & 3 & 0.0135 \\
\hline \multirow[t]{5}{*}{ Downregulated } & mmu04670:Leukocyte transendothelial migration & 16 & $1.83 \mathrm{E}-05$ \\
\hline & mmu04510:Focal adhesion & 20 & 7.17E-05 \\
\hline & mmu05210:Colorectal cancer & 12 & $2.15 \mathrm{E}-04$ \\
\hline & mmu04664:Fc epsilon RI signaling pathway & 11 & $6.15 \mathrm{E}-04$ \\
\hline & mmu04270:Vascular smooth muscle contraction & 13 & 0.0011 \\
\hline
\end{tabular}

KEGG, Kyoto Encyclopedia of Genes and Genomes; ECM, extracellular matrix.

processes, such as ribonucleoprotein complex biogenesis and ribosome biogenesis. Additionally, genes enriched in the greencolored module were significantly correlated with cell adhesion and biological adhesion. Moreover, both the dark green- and green-colored modules were significantly enriched for biosyn- thetic processes, such as water-soluble vitamin biosynthetic process and cholesterol biosynthetic process.

PPI network analysis. The PPI networks upon the DEGs in the dark green- and green-colored modules are shown 

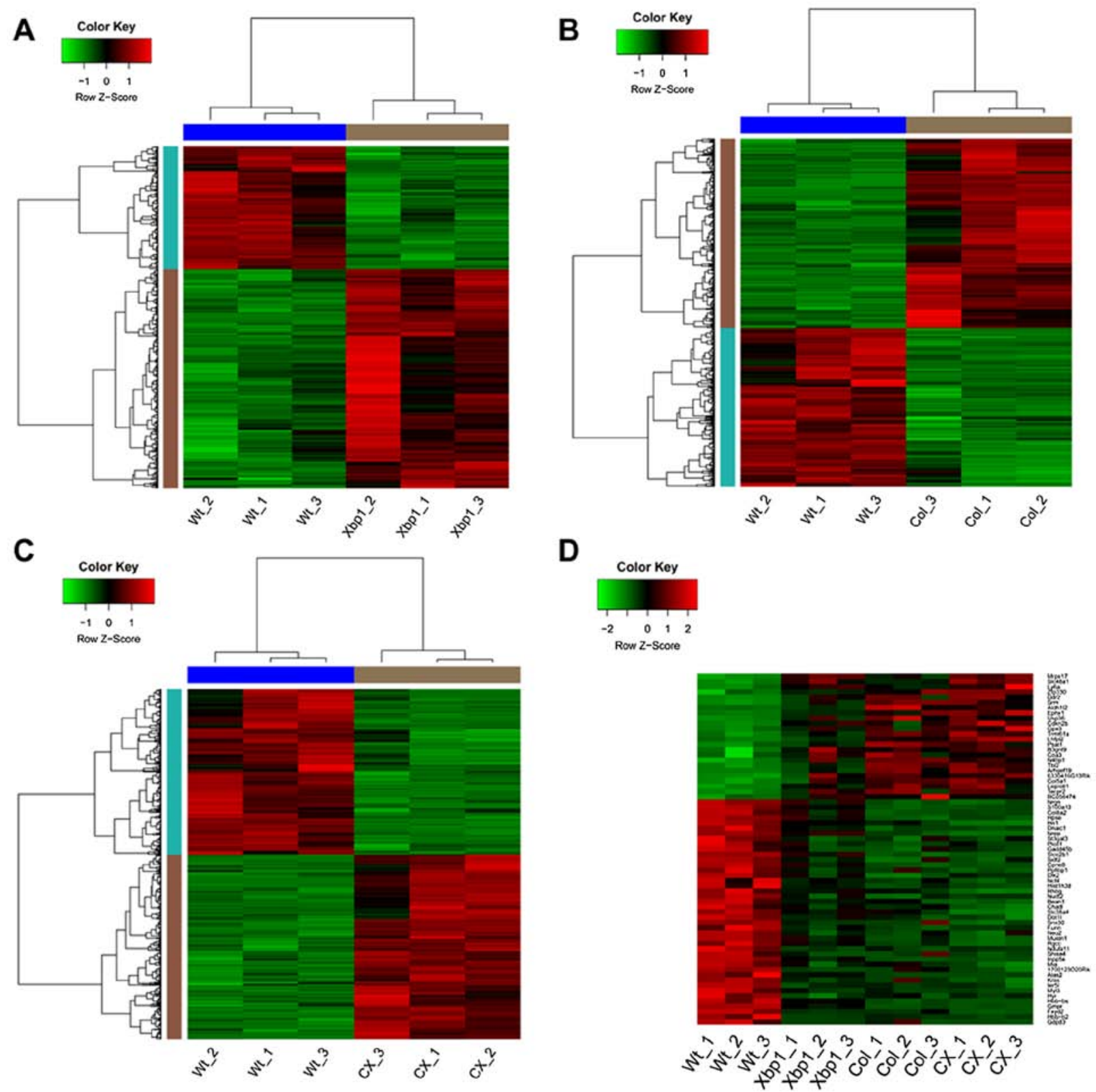

Figure 1. Heatmaps. (A) Heatmaps of differentially expressed genes (DEGs) identified in Xbp1 vs. WT. (B) Heat maps of DEGs identified in Col vs. WT. (C) Heat maps of DEGs identified in CX vs. WT. (D) Heat maps of overlapping DEGs in all the samples. Red color and green color represent a high expression level and low expression level of specific genes, respectively.

Table II. Results of gene co-expression module analysis.

\begin{tabular}{lcrl}
\hline Module & Correlation & Gene count & \multicolumn{1}{c}{$\mathrm{p}$-value } \\
\hline Turquoise & -0.6 & 503 & 0.03850043 \\
Dark orange & 0.17 & 40 & 0.590624 \\
Dark red & 0.37 & 28 & 0.2373936 \\
Dark olive green & 0.024 & 24 & 0.9413225 \\
Dark green & 0.94 & 378 & $4.28 \mathrm{E}-06$ \\
Pale turquoise & 0.69 & 31 & 0.01377187 \\
Black & 0.62 & 896 & 0.03280841 \\
Dark magenta & -0.78 & 10 & 0.002588195 \\
Green & -0.89 & 267 & $8.83 \mathrm{E}-05$ \\
Royal blue & -0.73 & 66 & 0.006466701 \\
Saddle brown & -0.35 & 15 & 0.2660367 \\
\hline
\end{tabular}

in Figs. 3 and 4, respectively. The PPI network of genes in the dark green-colored module consisted of 228 nodes and 444 interactions (edges) (Fig. 3). There were 13 DEGs with a node degree $>12$ in the network, namely, MRNA turnover 4 homolog $($ S. cerevisiae $)($ Mrto4) $($ degree $=27)$, ribosomal protein S27a $($ Rps27A) (degree $=25)$, WD repeat domain 74 $(W d r 74)($ degree $=21)$, FBJ murine osteosarcoma viral oncogene homolog $(F o s)($ degree $=20)$, vascular endothelial growth factor A $(V e g f a)($ degree $=19)$, IMP3, U3 small nucleolar ribonucleoprotein $($ Imp3) (degree $=17)$, Jun D proto-oncogene $($ Jund $)($ degree $=15)$, PWP2 periodic tryptophan protein homolog (yeast; $P w p 2)($ degree $=15)$, pescadillo ribosomal biogenesis factor $1($ Pes 1$)($ degree $=15)$, ribosomal RNA processing 9, small subunit ( $\mathrm{Ssu}$ ) processome component, homolog $($ yeast; $\operatorname{Rrp} 9)($ degree $=13)$, NOP2/Sun RNA 
Table III. The most significant GO terms of DEGs identified in darkgreen and green modules.

\begin{tabular}{|c|c|c|c|}
\hline Module & Term & $\mathrm{p}$-value & Gene \\
\hline \multirow[t]{10}{*}{ Darkgreen } & GO:0022613 - ribonucleoprotein complex biogenesis & 10 & 8.91E-04 \\
\hline & GO:0042254 - ribosome biogenesis & 9 & $9.96 \mathrm{E}-04$ \\
\hline & GO:0007050 - cell cycle arrest & 6 & 0.0037 \\
\hline & GO:0042364 - water-soluble vitamin biosynthetic process & 4 & 0.0071 \\
\hline & GO:0034470 - ncRNA processing & 9 & 0.0083 \\
\hline & GO:0000041 - transition metal ion transport & 6 & 0.0088 \\
\hline & GO:0055114 - oxidation reduction & 22 & 0.0109 \\
\hline & GO:0034660 - ncRNA metabolic process & 10 & 0.0116 \\
\hline & GO:0016053 - organic acid biosynthetic process & 8 & 0.0146 \\
\hline & GO:0046394 - carboxylic acid biosynthetic process & 8 & 0.0146 \\
\hline \multirow[t]{8}{*}{ Green } & GO:0007155 - cell adhesion & 14 & 0.0126 \\
\hline & GO:0022610 - biological adhesion & 14 & 0.0128 \\
\hline & GO:0006928 - cell motion & 10 & 0.0258 \\
\hline & GO:0006695 - cholesterol biosynthetic process & 3 & 0.0283 \\
\hline & GO:0055114 - oxidation reduction & 14 & 0.0456 \\
\hline & GO:0016126 - sterol biosynthetic process & 3 & 0.0462 \\
\hline & GO:0006865 - amino acid transport & 4 & 0.0482 \\
\hline & GO:0006694 - steroid biosynthetic process & 4 & 0.0482 \\
\hline
\end{tabular}

GO, Gene Ontology; DEGs, differentially expressed genes.

A

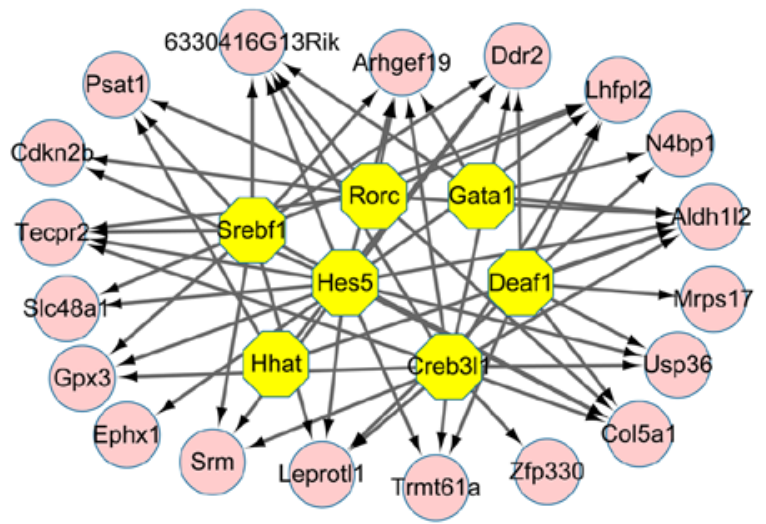

B

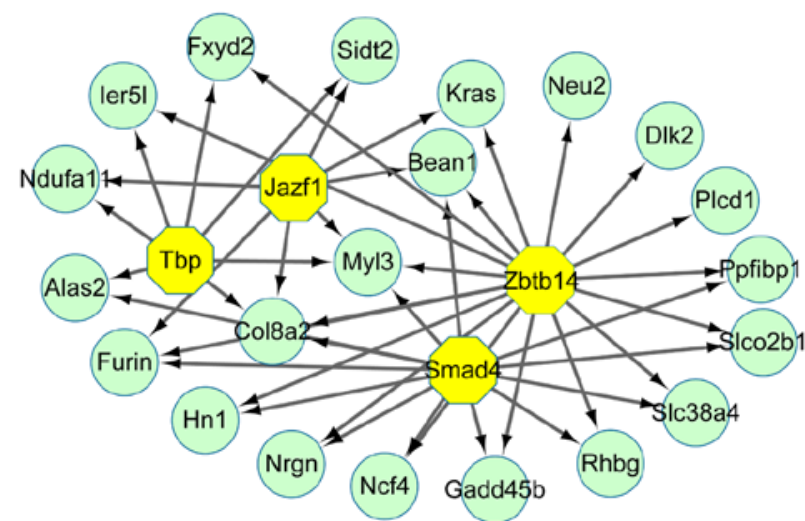

C

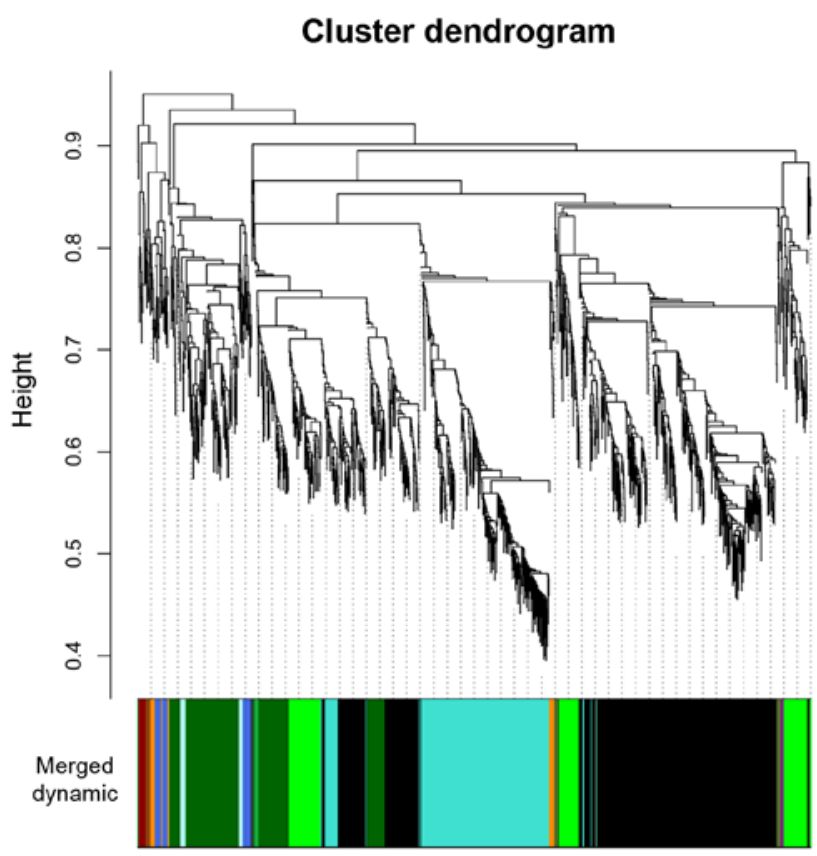

Figure 2. Regulatory networks and results of weighted correlation network analysis (WGCNA) analysis. (A) The regulatory network based on 7 transcriptional factors (TFs) and 19 common upregulated genes identified. The pink nodes indicate the common upregulated genes. The yellow nodes represent the TFs. (B) The regulatory network based on 4 TFs and 21 common downregulated genes identified. The green nodes indicate the common downregulated genes. The yellow nodes represent the TFs. (C) Gene dendrogram obtained by WGCNA. Each colored row underneath the dendrogram represents a color-coded module that contains a group of highly connected genes. 


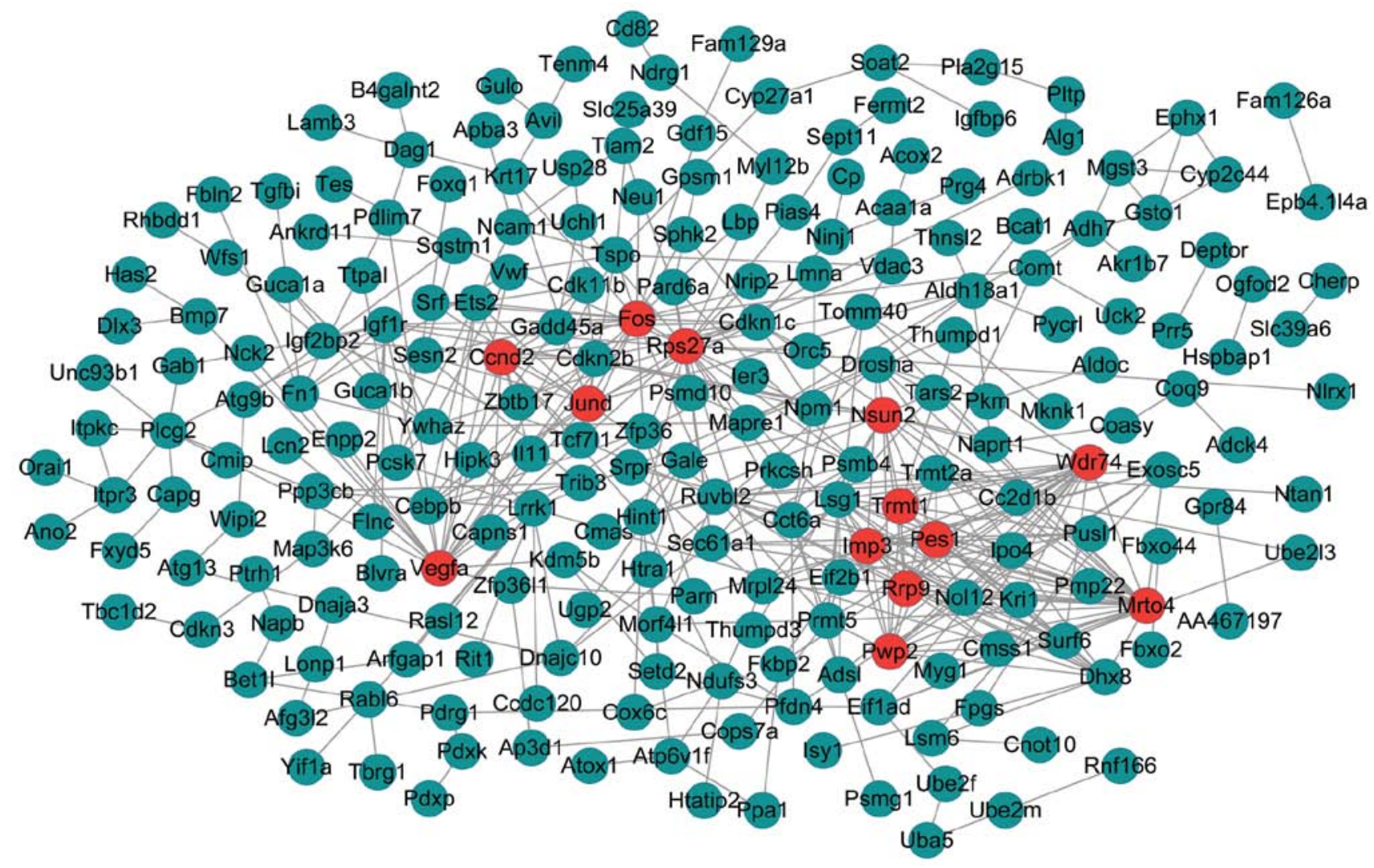

Figure 3. Protein-protein interaction (PPI) network of genes in the dark green-colored module. Nodes indicate genes.

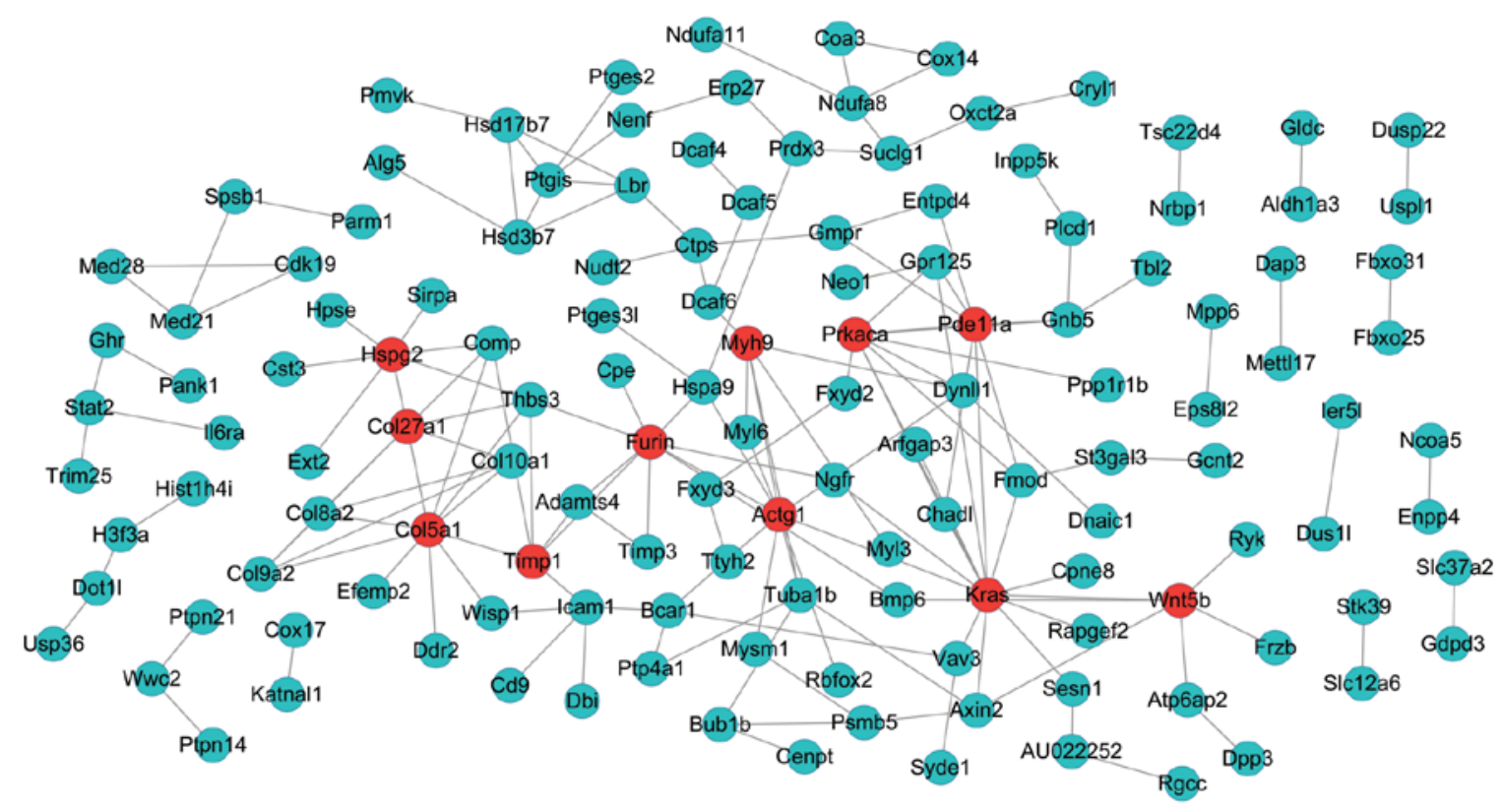

Figure 4. Protein-protein interaction (PPI) network of genes in the green-colored module. Nodes indicate genes.

methyltransferase family, member $2($ Nsun2) $($ degree $=13)$, TRNA methyltransferase 1 homolog (S. cerevisiae) (Trmt1) $($ degree $=13)$, cyclin D2 $($ Ccnd2 $)($ degree $=13)$. In addition, the 13 DEGs had no overlap in the regulatory networks.

On the other hand, the PPI network of genes in the green-colored module included 135 nodes and 166 interactions (edges) (Fig. 4). Besides, there were 11 DEGs with a node degree $>5$ in this network, namely, Kirsten rat sarcoma viral oncogene homolog $($ Kras $)($ degree $=14)$, collagen, type V, alpha 1 (Col5al) (degree $=11)$, actin gamma $1(\operatorname{Actg} 1)($ degree $=10)$, protein kinase, CAMP-dependent, catalytic, alpha $($ Prkaca $)($ degree $=9)$, Furin (paired basic amino acid cleaving enzyme) (Furin) $($ degree $=9)$, heparan sulfate proteoglycan $2(H s p g 2)$ 
$($ degree $=8)$, phosphodiesterase 11A $($ Pde11A $)($ degree $=8)$, collagen, type XXVII, alpha 1 (Col27al) (degree $=7)$, wingless-type MMTV integration site family, member 5B $(W n t 5 b)($ degree $=6)$, myosin, heavy chain 9 , non-muscle $(M y h 9)$ (degree $=6)$, TIMP metallopeptidase inhibitor 1 (Timpl) (degree =6). Fortunately, we found that 3 of these 11 DEGs, Kras (downregulated), Col5al (upregulated), and Furin (upregulated) were also included in the regulatory networks.

\section{Discussion}

In the current study, 481, 1,530 and 1,214 DEGs were screened out in Xbp1 vs. WT, Col vs. WT and CX vs. WT, respectively. Pathway enrichment analysis revealed that these DEGs were enriched in different pathways, such as ECM-receptor interaction, focal adhesion and pathways associated with metabolism. In total, 7 TFs were found to regulate 19 common upregulated genes that were with consistent gene change in the 3 groups, while 4 TFs were identified to regulate 21 common downregulated genes. WGCNA demonstrated that 2 significantly enriched gene co-expression modules (dark green- and green-colored modules) and DEGs in the 2 modules were mainly enriched different biological processes, such as ribosome biogenesis. Moreover, Kras (downregulated), Col5al (upregulated), and Furin (upregulated) were both in the regulatory networks and PPI network.

With greater than 2-fold differential expression and with an adjusted p-value of 0.01, Cameron et al (11) identified 1,337, 215 and 1,633 differentially expressed probes in CX vs. WT, Xbpl vs. WT and Col vs. WT, respectively. The amount of DEGs identified in this study was slightly different from the original study of Cameron et al (11), which may result from different analysis methods or analytical errors. In the present study, we first paid more attention to the DEGs which had not been analyzed in the original study by Cameron et al (11), including DEGs in Col vs. WT and CX vs. WT and Xbpl vs. WT, as well as the DEGs between in Col vs. WT and Xbpl vs. WT, but not CX vs. WT. Finally, our focus was the DEGs both identified in the regulatory networks and PPI networks.

Kras was one of the DEGs that were identified in the regulatory networks and PPI network. Kras, a Kirsten ras oncogene homolog from the mammalian ras gene family, encodes a protein that is a member of the small GTPase superfamily (25). Evidence has indicated that Kras can orchestrate multiple metabolic changes, including differential channeling of glucose intermediates, stimulation of glucose uptake, and reprogrammed glutamine metabolism (26). Moreover, Chan et al demonstrated that intracellular mutant collagen $\mathrm{X}$ could lead to deregulated cellular metabolism (27). Taken together, we suggested that Kras may play a critical role in the development of SMCD via the regulation of cell metabolism.

It is well known that SMCD can be caused by heterozygous mutations in the CollOal gene (4). In the present study, Col5al was found to be another significant DEG identified both in the regulatory networks and PPI network. Col5al encodes an alpha chain for one of the low abundance fibrillar collagens (28). It has been demonstrated that Col5al is regulated by transforming growth factor- $\beta$ (TGF- $\beta$ ) in osteoblasts (29), and Col5al is involved in the collagen biosynthesis, which is associated with chondrocyte differentiation (11). In agreement with previous studies, we infer that Col5al may play a critical role in the pathogenesis of SMCD by participating in chondrocyte differentiation, although further verifications are required to confirm this result.

Furthermore, Furin was another focus in this study, which was identified both in the regulatory networks and PPI network. Furin is likely to represent the ubiquitous endoprotease activity within constitutive secretory pathways (30). Evidence has indicated that Furin is an authentic TGF- $\beta 1$-converting enzyme (30). Besides, pericellular and diffuse interterritorial distribution of TGF- $\beta 2$ has been observed in patients with SMCD and there may be a functional interaction of TGF- $\beta 2$ and type $\mathrm{X}$ collagen (31). Thus, Furin may be essential in the mechanisms of SMCD, which warrants further investigation.

However, this study has several limitations. First, a larger sample size in the further investigations is warranted in order to verify our findings. Second, the lack of cross-check and experimental verification were also limitations. Validation using other datasets in similar topic may be used to cross-check our results. In the future, we aim to carry out experimental verifications using different analytical approaches, such as reverse transcription-quantitative polymerase chain reaction (RT-qPCR) and immunohistochemistry.

In conclusion, the data of the present study revealed several potential key genes (Kras, Col5al and Furin) which may play a role in the molecular mechanisms responsible for SMCD. $K R A S$ may play a critical role in the development of SMCD via the regulation of cell metabolism. Besides, Col5al may play a critical role in the pathogenesis of SMCD by participating in chondrocyte differentiation. Furin may be essential to the mechanisms of SMCD through an interaction with type X collagen. Further characterization of the genes identified in this study may provide deeper insight into the pathology of SMCD.

\section{References}

1. Park H, Hong S, Cho SI, Cho TJ, Choi IH, Jin DK, Sohn YB, Park SW, Cho HH, Cheon JE, et al: Case of mild Schmid-type metaphyseal chondrodysplasia with novel sequence variation involving an unusual mutational site of the COL10A1 gene. Eur J Med Genet 58: 175-179, 2015

2. Bateman JF, Freddi S, Nattrass G and Savarirayan R: Tissue-specific RNA surveillance? Nonsense-mediated mRNA decay causes collagen X haploinsufficiency in Schmid metaphyseal chondrodysplasia cartilage. Hum Mol Genet 12: 217-225, 2003.

3. Patterson SE and Dealy CN: Mechanisms and models of endoplasmic reticulum stress in chondrodysplasia. Dev Dyn 243: 875-893, 2014.

4. Bogin O, Kvansakul M, Rom E, Singer J, Yayon A and Hohenester E: Insight into Schmid metaphyseal chondrodysplasia from the crystal structure of the collagen $\mathrm{X} \mathrm{NC1}$ domain trimer. Structure 10: 165-173, 2002.

5. Woelfle JV, Brenner RE, Zabel B, Reichel H and Nelitz M: Schmid-type metaphyseal chondrodysplasia as the result of a collagen type $\mathrm{X}$ defect due to a novel COL10A1 nonsense mutation: A case report of a novel COL10A1 mutation. J Orthop Sci 16: 245-249, 2011.

6. Warman ML, Cormier-Daire V, Hall C, Krakow D, Lachman R, LeMerrer M, Mortier G, Mundlos S, Nishimura G, Rimoin DL, et al: Nosology and classification of genetic skeletal disorders: 2010 revision. Am J Med Genet A 155A: 943-968, 2011.

7. Arnold WV and Fertala A: Skeletal diseases caused by mutations that affect collagen structure and function. Int J Biochem Cell Biol 45: 1556-1567, 2013. 
8. Hetz C: The unfolded protein response: Controlling cell fate decisions under ER stress and beyond. Nat Rev Mol Cell Biol 13: 89-102, 2012.

9. Rajpar MH, McDermott B, Kung L, Eardley R, Knowles L, Heeran M, Thornton DJ, Wilson R, Bateman JF, Poulsom R, et al: Targeted induction of endoplasmic reticulum stress induces cartilage pathology. PLoS Genet 5: e1000691, 2009.

10. Yoshida $\mathrm{H}$, Matsui T, Yamamoto A, Okada T and Mori K: XBP1 mRNA is induced by ATF6 and spliced by IRE1 in response to ER stress to produce a highly active transcription factor. Cell 107: 881-891, 2001.

11. Cameron TL, Bell KM, Gresshoff IL, Sampurno L, Mullan L, Ermann J, Glimcher LH, Boot-Handford RP and Bateman JF: XBP1-Independent UPR Pathways Suppress C/EBP- $\beta$ Mediated Chondrocyte Differentiation in ER-Stress Related Skeletal Disease. PLoS Genet 11: e1005505, 2015.

12. Bolstad BM: preprocessCore: A collection of pre-processing functions. R package version 1: 2013. http://www.bioconductor or $\mathrm{g} / \mathrm{packages} / \mathrm{release} / \mathrm{bioc} / \mathrm{html} / \mathrm{preprocessCore.html}$. Accessed March 27, 2013.

13. Gardeux V, Arslan AD, Achour I, Ho TT, Beck WT and Lussier YA: Concordance of deregulated mechanisms unveiled in underpowered experiments: PTBP1 knockdown case study. BMC Med Genomics 7 (Suppl 1): S1, 2014.

14. Hoeksema MA, Scicluna BP, Boshuizen MC, van der Velden S, Neele AE, Van den Bossche J, Matlung HL, van den Berg TK, Goossens $\mathrm{P}$ and de Winther MP: IFN- $\gamma$ priming of macrophages represses a part of the inflammatory program and attenuates neutrophil recruitment. J Immunol 194: 3909-3916, 2015.

15. Ritchie ME, Phipson B, Wu D, Hu Y, Law CW, Shi W and Smyth GK: limma powers differential expression analyses for RNA-sequencing and microarray studies. Nucleic Acids Res 43 . e47, 2015 .

16. Warnes GR, Bolker B, Bonebakker L, Gentleman R, Huber W, Liaw A, Lumley T, Maechler M, Magnusson A and Moeller S: gplots: Various R programming tools for plotting data. R package version 2.12. 1. 2013. http://cran.r-project.org/web/packages/ gplots/index.html.

17. Dennis G Jr, Sherman BT, Hosack DA, Yang J, Gao W, Lane HC and Lempicki RA: DAVID: Database for annotation, visualization, and integrated discovery. Genome Biol 4: 3, 2003.

18. Mao X, Cai T, Olyarchuk JG and Wei L: Automated genome annotation and pathway identification using the KEGG Orthology (KO) as a controlled vocabulary. Bioinformatics 21 : 3787-3793, 2005.

19. Janky R, Verfaillie A, Imrichová H, Van de Sande B, Standaert L, Christiaens V, Hulselmans G, Herten K, Naval Sanchez M, Potier D, et al: iRegulon: from a gene list to a gene regulatory network using large motif and track collections. PLoS Comput Biol 10: e1003731, 2014.
20. Smoot ME, Ono K, Ruscheinski J, Wang P-L and Ideker T: Cytoscape 2.8: New features for data integration and network visualization. Bioinformatics 27: 431-432, 2011.

21. Langfelder P and Horvath S: WGCNA: An R package for weighted correlation network analysis. BMC Bioinformatics 9: $559,2008$.

22. Franceschini A, Szklarczyk D, Frankild S, Kuhn M, Simonovic M, Roth A, Lin J, Minguez P, Bork P, von Mering C, et al: STRING v9.1: Protein-protein interaction networks, with increased coverage and integration. Nucleic Acids Res 41: D808-D815, 2013.

23. Szklarczyk D, Franceschini A, Kuhn M, Simonovic M, Roth A, Minguez P, Doerks T, Stark M, Muller J, Bork P, et al: The STRING database in 2011: Functional interaction networks of proteins, globally integrated and scored. Nucleic Acids Res 39: D561-D568, 2011.

24. Spinelli L, Gambette P, Chapple CE, Robisson B, Baudot A, Garreta H, Tichit L, Guénoche A and Brun C: Clust\&See: A Cytoscape plugin for the identification, visualization and manipulation of network clusters. Biosystems 113: 91-95, 2013.

25. Vakiani E and Solit DB: KRAS and BRAF: Drug targets and predictive biomarkers. J Pathol 223: 219-229, 2011.

26. Bryant KL, Mancias JD, Kimmelman AC and Der CJ: KRAS: Feeding pancreatic cancer proliferation. Trends Biochem Sci 39: 91-100, 2014

27. Chan D, Ho MS and Cheah KS: Aberrant signal peptide cleavage of collagen $\mathrm{X}$ in Schmid metaphyseal chondrodysplasia. Implications for the molecular basis of the disease. J Biol Chem 276: 7992-7997, 2001.

28. Raleigh SM, van der Merwe L, Ribbans WJ, Smith RK, Schwellnus MP and Collins M: Variants within the MMP3 gene are associated with Achilles tendinopathy: Possible interaction with the COL5A1 gene. Br J Sports Med 43: 514-520, 2009.

29. Kahai S, Vary CP, Gao Y and Seth A: Collagen, type V, $\alpha 1$ (COL5A1) is regulated by TGF- $\beta$ in osteoblasts. Matrix Biol 23 445-455, 2004

30. Dubois CM, Blanchette F, Laprise MH, Leduc R, Grondin F and Seidah NG: Evidence that furin is an authentic transforming growth factor- $\beta 1$-converting enzyme. Am J Pathol 158: 305-316, 2001.

31. Yang M, Wang X, Zhang L, Yu C, Zhang B, Cole W, Cavey G, Davidson $P$ and Gibson G: Demonstration of the interaction of transforming growth factor beta 2 and type $\mathrm{X}$ collagen using a modified tandem affinity purification tag. J Chromatogr B Analyt Technol Biomed Life Sci 875: 493-501, 2008. 